OPEN ACCESS

Edited by:

Haina Shin,

Washington University in St. Louis,

United States

Reviewed by:

Maria Tokuyama

University of British Columbia, Canada

Kristina Eriksson,

University of Gothenburg, Sweden

*Correspondence:

Ali A. Ashkar

ashkara@mcmaster.ca

tThese authors have contributed equally to this work and share first authorship

Specialty section: This article was submitted to Viral Immunology, a section of the journal

Frontiers in Immunology

Received: 13 June 2021 Accepted: 02 August 2021 Published: 18 August 2021

Citation:

Feng E, Balint E, Vahedi F and Ashkar AA (2021) Immunoregulatory

Functions of Interferons

During Genital HSV-2 Infection.

Front. Immunol. 12:724618. doi: 10.3389/fimmu.2021.724618

\section{Immunoregulatory Functions of Interferons During Genital HSV-2 Infection}

\author{
Emily Feng ${ }^{\dagger}$, Elizabeth Balint ${ }^{\dagger}$, Fatemah Vahedi and Ali A. Ashkar* \\ McMaster Immunology Research Centre, Department of Medicine, McMaster University, Hamilton, ON, Canada
}

Herpes simplex virus type 2 (HSV-2) infection is one of the most prevalent sexually transmitted infections that disproportionately impacts women worldwide. Currently, there are no vaccines or curative treatments, resulting in life-long infection. The mucosal environment of the female reproductive tract (FRT) is home to a complex array of local immune defenses that must be carefully coordinated to protect against genital HSV-2 infection, while preventing excessive inflammation to prevent disease symptoms. Crucial to the defense against HSV-2 infection in the FRT are three classes of highly related and integrated cytokines, type I, II, and III interferons (IFN). These three classes of cytokines control HSV-2 infection and reduce tissue damage through a combination of directly inhibiting viral replication, as well as regulating the function of resident immune cells. In this review, we will examine how interferons are induced and their critical role in how they shape the local immune response to HSV-2 infection in the FRT.

Keywords: HSV - 2, immune regulation, female reproductive tract (FRT), type I interferon (IFN), type II interferon, type III interferons, genital mucosa

\section{INTRODUCTION}

Genital herpes simplex virus type 2 (HSV-2) infection remains one of the most prevalent sexually transmitted infections, with an estimated 491.6 million cases worldwide (1). African cohorts comprise of the majority of the cases, with $43.9 \%$ and $25.4 \%$ seropositivity in women and men respectively, and HSV-2 disproportionately impacts women in African cohorts and globally (1). Despite the prevalence of HSV-2 infection, no preventative or curative treatments have been developed. Current treatments for HSV-2 infection only involve antivirals to suppress reactivation, but HSV-2 remains a life-long infection. Unfortunately, resistance to antivirals such as acyclovir, particularly in immunocompromised individuals, has been observed (2).

Genital HSV-2 infection in the vaginal mucosa begins at lytic replication in keratinocytes of the epithelial lining, resulting in genital lesions (3). HSV-2 is also a neurotropic virus and can infect the brain and result in fatal herpes simplex encephalitis in newborns or immunocompromised individuals $(4,5)$. Largely, HSV-2 infections are self-limiting, and are often asymptomatic. However, asymptomatic individuals still frequently shed the virus during reactivation periods (68). Those that experience symptomatic infection will often experience reactivation of genital lesions throughout their lifetime (9). While antivirals, such as acyclovir, can reduce the frequency of HSV reactivation, they cannot eliminate or completely prevent viral shedding (9). Furthermore, individuals with genital HSV-2 infection also hold a significantly increased risk of acquiring 
HIV-1 infection, partially through impaired integrity of the vaginal mucosal barrier due to genital lesions $(10,11)$. Thus, the inability to adequately address the HSV-2 epidemic has also been driving the HIV epidemic.

Regulation of innate and adaptive immune responses in the genital mucosa is critical in both the control of HSV-2 infection and reactivation, as well as inhibiting the development of genital and neural tissue pathology. Interferons (IFN), including classes I, II, and III, are well known for their antiviral functions, but their role in immune regulation is slowly coming to light. The critical importance of IFNs is best evidenced by the multiple mechanisms of evasion of IFN-induced responses by the HSV2 virion to evade IFN induction and signaling pathways (12). Understanding how immunity to HSV-2 infections is regulated by IFNs remains a crucial piece in developing better therapeutics and an effective vaccine for genital herpes infections. In this review, we will describe the various mechanisms of IFN-mediated regulation of innate and adaptive immunity to genital HSV-2 infection, emphasizing its ability to prevent the development of pathogenic immunity.

\section{INDUCTION AND REGULATION OF IFN RESPONSES TO GENITAL HSV-2 INFECTION}

Type I IFNs are a class of signaling molecules that include most notably IFN- $\alpha$ and its subtypes, IFN- $\beta$, as well as lesser understood IFN- $\epsilon$, IFN- $\omega$, and IFN- $\kappa$ (13). The induction of type I IFNs during genital HSV-2 infection contributes significantly to resistance to infection, through both directly suppressing viral replication and facilitating antiviral immune responses $(14,15)$. Type I IFNs signal through their dimeric interferon $\alpha / \beta$ receptor (IFNAR) to induce a JAK/STAT signaling cascade that includes the transcription of interferonstimulated genes (ISGs) to inhibit viral replication (13). Induction of type I IFN occurs through DNA sensing pattern recognition receptors, including endosomal toll like receptor 9 (TLR9), and cytosolic sensors IFI16 and cyclic GMP-AMP synthase (cGAS) stimulating the STING adaptor protein (16, 17). Interestingly, Eriksson et al. demonstrated that polymorphisms in IFI16 impacting expression levels correlated with both IFN- $\beta$ production and susceptibility to genital HSV-2 infection (18). The administration of both TLR agonists and STING agonists in mice have demonstrated to protect against genital herpes infection and potently induce type I IFNs $(14,19)$. Recognition of replication intermediate dsRNA has been shown by retinoic acid-inducible gene-like receptors (RLRs) RIG-I and MDA5 (16, 17). IFN-€, produced at canonically low levels in the reproductive mucosa, is not induced through typical TLR or RIG-I signaling pathways (20).

IFN- $\alpha / \beta$ is primarily produced by circulating plasmacytoid dendritic cells (pDCs) through recognition by TLR9 during genital HSV-2 infection (16, 21, 22). Genital lesions in recurrent $\mathrm{HSV}-2$ patients display $\mathrm{pDC}$ infiltration, suggesting a role of type I IFNs in controlling HSV-2 reactivation (23).
However, others have demonstrated that pDCs are only involved in systemic infections in mice, and not local mucosal infections (24). These studies have also credited classical CD8 $\alpha$ DCs as a major source of IFN production, independent of TLR9 signaling $(22,24)$. On the other hand, unlike IFN- $\alpha / \beta$, IFN- $€$ was found to be constitutively expressed by epithelial cells in the uterus and ovaries of both mice and humans (20). Together, these studies show that the production of type I IFNs is critical to inducing protective innate and adaptive immune responses in response to genital HSV-2 infections.

Type II IFNs consist only of one subtype, IFN- $\gamma$. The induction of IFN- $\gamma$ is strongly dependent on type I IFN signaling. Type II IFNs are largely produced from NK cells and T cells during genital HSV-2 infection, stimulated by type I IFNmediated IL-18 signaling (25). IFN- $\gamma$ signaling occurs through the IFN- $\gamma$ receptor present on the majority of immune cells (13). Several studies have identified a critical role of IFN- $\gamma$ in mediating protection against genital $\mathrm{HSV}-2$ infection. In vitro HSV-2 infection of human PBMCs demonstrates IFN- $\gamma$ as the predominant cytokine produced, and dysregulated IFN- $\gamma$ production is associated with recurrent genital herpes in humans $(26,27)$. This dysregulation has been associated with genetic variations in the STAT4 gene, which regulates IFN- $\gamma$ production (28). Individuals with recurrent disease also exhibit impaired cellular responses to IFN- $\gamma$ signaling, as treatment of HSV-2-infected macrophages from these individuals with IFN- $\gamma$ enhanced viral replication (26). Likewise, several mouse models have demonstrated that IFN- $\gamma$ is required for HSV-2 clearance during primary and secondary challenge (28-33).

Type III IFNs have emerged as another family of IFNs with a critical role in the induction of antiviral responses to HSV-2 infection. Type III IFNs, consisting of IFN- $\lambda 1,2,3$, and 4 in humans, and functional IFN- $\lambda 2$ and 3 in mice, have already been established as potent inhibitors of viral replication $(34,35)$. DCs and pDCs are a primary producer of type III IFNs in response to TLR7 ligands and HSV-2 infection (36-38). However, most cells can produce type III IFNs, including mucosal epithelial cells. Type III IFNs are similar to the type I IFN canonical signaling pathway, but differ in their effector cells $(35,39)$. The expression of the IFN- $\lambda$ heterodimeric receptor, IL-28R $\alpha / \mathrm{IL}-10 \mathrm{R} \beta$, is more restricted than IFNAR, as it is highly expressed on mucosal epithelial cells in the genital mucosa (40-43). Some evidence suggests that the type III IFN receptor is not expressed on the surfaces of immune cells, leading to its less inflammatory responses (44); however, this has been increasingly challenged, with emerging evidence suggesting type III IFNs may possess direct immunoregulatory functions.

Susceptibility to HSV-2 infection has been suggested to be influenced by hormone-mediated alterations in IFN responses. In mice, susceptibility to intravaginal HSV-2 infection is dependent on treatment with progesterone contraceptive hormones to induce diestrus, a state of low estrogen levels (45). Estradiol treatment in women increased capacity to produce type I IFN by pDCs following just one month of treatment (46). IFN- $\epsilon$ is potentially even more strongly regulated by hormone levels, with lowest levels during diestrus 
and highest production during estrus, and IFN- $\epsilon$ deficient mice display increased susceptibility to HSV-2 infection (20). Meanwhile, progesterone signaling not only increases susceptibility to mouse HSV-2 infection, but impedes type I IFN responses $(45,47,48)$. Depot medroxyprogesterone acetate (DMPA) treatment impairs TLR9 ligand-mediated IFN- $\alpha$ production by inhibiting IRF7 nuclear accumulation following CpG stimulation in both human and mouse pDCs $(47,49)$. These findings strongly emphasize the importance of understanding the impact of hormones, from either biological factors or from contraceptives, on the development of protective immunity to HSV-2 infection, and its considerations on therapeutic development. Moreover, this highlights the dependency on type I IFNs for protection against HSV-2 infection.

\section{THE ROLE OF INTERFERONS IN REGULATION OF INNATE IMMUNITY DURING HSV-2 INFECTION}

Innate immunity in the genital mucosa plays a critical role in initial viral infection and replication, however their dysregulation can also be the cause of severe inflammation and tissue damage. In this section, we will explore the innate immune responses regulated by IFNs towards genital HSV-2 infection.

\section{Monocytes/Macrophages}

During HSV-2 infection, monocyte recruitment is crucial for controlling viral infection and inducing antiviral immunity in the vaginal mucosa (50). Monocytes/macrophages upregulate the expression of Fas and FasL during infection (51). Although typically involved in inducing the apoptosis of infected cells, HSV-2 infected keratinocytes in a mouse model displayed resistance to Fas/FasL-mediated apoptosis, but strong production of inflammatory cytokines, including TNF- $\alpha$, IL- $1 \beta$, and CXCL1/2 (52). Infected monocytes in mice, while susceptible to Fas-mediated apoptosis, also respond with the production of inflammatory cytokines and CXCL9/10 T cell chemokines (51).

Evidence has suggested that type I IFN signaling may regulate the protective effects of monocyte/macrophages during HSV-2 infection. Type I IFN has been demonstrated to induce FasL expression during influenza infection in mice on immune cells (53), though this has not been defined during HSV-2 genital infection. Type I IFN regulation of Fas/FasL pathways during HSV-2 infection would promote monocyte-mediated inflammation and the induction and recruitment of adaptive immune responses. Type I IFN-mediated recruitment of inflammatory monocytes (IM) during infection has been clearly defined in murine HSV-1 and HSV-2 infection, promoting survival and antiviral responses $(50,54)$. The immune stimulatory effect on monocytes is dependent on type I IFN-mediated production of CCL2 during HSV-2 infection that promotes recruitment to the genital mucosa $(25,55)$. Therefore, type I IFN signaling during HSV-2 infection promotes both the recruitment of inflammatory monocytes to the genital mucosa, but also Fas/FasL-induced inflammation in the clearance of infection.
Type II IFNs produced by NK cells and T cells also promote macrophage responses during HSV-2 infection. Macrophage nitric oxide production is stimulated by IFN- $\gamma$ signaling, and HSV-2infected mouse macrophages exhibit enhanced nitric oxide release upon IFN- $\gamma$ stimulation (56). IFN- $\gamma$ is a Th1 promoting cytokine shown to promote macrophage M1 polarization and production of pro-inflammatory cytokines, as well as upregulation of MHC class II expression to facilitate effective adaptive immune responses in a mouse model of HSV -2 infection $(57,58)$. Overall, type I and II IFNs facilitate the recruitment and activation of antiviral functions of both IM and macrophages during HSV-2 infection.

\section{Neutrophils}

Neutrophils can play a protective role in HSV-2 infection, but their dysregulation can lead to damaging inflammatory outcomes. Early neutrophil recruitment to the genital mucosa can limit the establishment of HSV-2 infection in mice (59). IL-36 $\gamma$ production by mucosal epithelial cells in HSV-2 infection suppressed viral replication through neutrophil recruitment and expression of neutrophil chemokines CXCL1 and CXCL2 (60). This in turn prevented viral dissemination, and importantly, neuroinvasion. Interestingly, IL-36 $\gamma$ induction has been demonstrated to increase sensitivity to IFN- $\alpha / \beta$ during HSV-2 infection in mice through increased IFNAR expression on keratinocytes, likely also playing a role in the protective function of IL-36 (61).

However, despite the role of neutrophils in limiting early viral replication, neutrophils have often been demonstrated to cause damaging inflammatory immune responses and are implicated in increasing disease severity during viral infection $(62,63)$. Similarly, neutrophils have been described to drive liver damage in a murine model for systemic HSV-2 infection (64). These pathogenic effects of neutrophils are strongly regulated by type I IFN signaling. While CXCL1 is upregulated in vitro following HSV-2 infection, type IIFN signaling can suppress the expression of neutrophil chemokines by IM and reduce their recruitment to the sensory ganglia during mouse HSV-1 infection and other mucosal infections, such as IAV $(62,65)$. This process of IFN-mediated regulation of neutrophils would inhibit HSV-2 infection-induced neuronal damage. Similarly, IFN- $\lambda$ has been shown to suppress neutrophilmediated damage in mice in the intestinal mucosa, though this has yet to be confirmed in the genital mucosa (66). On the contrary, dysregulated and prolonged type I IFN signaling was recently described to promote epithelial damage in response to mouse HSV infection by neutrophils (67). Nonetheless, early type I IFN signaling in this model is still important for viral control and survival of HSV-2 infection (67). In conclusion, we observe that regulated type I IFN responses during HSV-2 infection promotes protective neutrophil functions while also suppressing pathogenic immune activation in the central nervous system (CNS).

\section{NK Cells}

It has been well established that Natural Killer (NK) and NKT cells are critically required for innate protection against HSV-2 infections, and cases of individuals with severe NK cell deficiencies have been associated with recurrent HSV infections (68). NK cell-deficient mouse models are highly 
susceptible to HSV-2 infection, with greater viral load and mortality (69). Likewise, loss of NK cell recruitment by chemokines CXCL9 and CXCL10, or loss of the CCR5 receptor, increased susceptibility to intravaginal HSV-2 infection in mice $(70,71)$. Impairment of NK cell recruitment increased viral load in both the genital mucosa and the central nervous system, highlighting its critical role in suppressing local viral replication as well as neuroinvasion $(70,71)$.

Type I IFN production during infection is essential for NK cell activation and has long been suggested to activate NK cells (72-75). Furthermore, NK cell memory has been observed in mouse HSV-2 infection, and type I IFN signaling promotes NK cell expansion, protection against fratricide, and induction of $\mathrm{NK}$ cell memory (76-78). However, we and others have shown that type I IFNs induce NK cell activation through an indirect mechanism. Loss of IFNAR in a mouse model of HSV-2 infection suppressed IFN- $\gamma$ production by NK cells, but not $\mathrm{NK}$ cell recruitment to vaginal tissue at 2 days post-infection, suggesting a role in NK cell activation but not recruitment (79). In response to systemic and local infection, as well as TLR stimulation, type I IFN signaling on DCs is known to induce IL-15 trans-presentation to NK cells recruited to lymph nodes, as well as increased IL-15R $\alpha$ expression (79). However, absence of type I IFN signaling in a mouse model of HSV-2 genital infection resulted in enhanced IL-15 production, with no impacts on IL-15R $\alpha$ expression $(25,72)$. Thus, impaired NK cell activation in the absence of type I IFN signaling is not mediated by reduced IL-15 trans-presentation.

During HSV-2 infection, IMs are key mediators of NK cell activation. Type I IFN signaling via IFNAR on IM induces the production of the cytokine IL-18 typically capable of inducing NK cell IFN- $\gamma$ production (25). IFN- $\lambda 3$ may also induce NK cell activity through direct signaling on monocyte-macrophages and their production of NK cell stimulatory cytokines, as seen in influenza infection in mice (80). While IL-12 and IL-18 are well-known to synergize for optimal NK cell IFN- $\gamma$ production, IM production of IL-18 was critical for NK cell activation in the context of HSV-2 infection (25, 81-83). In Ifnar ${ }^{-/-}$and $\mathrm{Il}_{-18^{-/-}}$ mice, reduced IL-18 production resulted in a complete lack of IFN- $\gamma$ production during early HSV-2 infection (25). This was specifically due to a lack of $\mathrm{NK}$ cell activation, as $\mathrm{NK}$ cell recruitment was not affected by IFNAR and IL-18 deficiency. Overall, type I IFNs are critical mediators of indirect NK cell activation and IFN- $\gamma$ production during mouse HSV- 2 infection.

In addition to its role in the induction of adaptive immunity and type II IFN production, type I IFN has also been suggested to possess immunoregulatory properties for NK cells. Interestingly, direct type I IFN signaling on both human and mouse NK cells inhibits NK cell IFN- $\gamma$ production (84). Excessive IFN- $\gamma$ production has been shown to promote immune-mediated tissue damage. For example, high production of IFN- $\gamma$ in the central nervous system can lead to microglia-mediated demyelination and neurological sequelae in mice $(85,86)$. Similarly, IFN- $\gamma$ production during mouse HSV-2 infection is tightly regulated by type I IFNs to prevent excessive immune activation and immunopathology, peaking at 2 days post-infection (dpi) and rapidly decreasing by 3 dpi (84). Thus, type I IFN signaling during HSV-2 infection critically regulates both the induction and control of NK cell-mediated type II IFN signaling.

\section{THE ROLE OF INTERFERONS IN REGULATION OF ADAPTIVE IMMUNITY DURING HSV-2 INFECTION}

As we have discussed, induction of effective and appropriate innate immune responses is critical for early protection against HSV-2 infection, as well as restricting immune-mediated damage. However, IFNs are also necessary to direct an appropriate adaptive immune response and develop immune memory. This section will clarify the mechanisms through which IFNs promote Th1 adaptive immune responses and vaccineinduced memory while preventing harmful, suboptimal $\mathrm{T}$ and $\mathrm{B}$ cell responses.

\section{Dendritic Cells Mediate Innate/Adaptive Crosstalk}

To establish an appropriate adaptive immune response to HSV-2 infection, early antiviral responses via type I IFN signaling are required. The FRT consists of various populations of antigen presentating cells (APCs), including Langerhans cells in the epithelium, submucosal DCs, and monocyte-derived DCs (87). As previously described, IFNs are necessary for the recruitment of IM to the vaginal tract. Iijima et al. demonstrated that type I IFN signaling during HSV-2 infection in mice is required to recruit IM via CCR2, and that IM-derived DCs play a distinct role in restimulation of effector Th1 $\mathrm{CD}^{+}{ }^{+} \mathrm{T}$ cells to produce IFN- $\gamma(50)$. Type I IFNs have also been demonstrated to induce DC maturation and DC-facilitated Th1 responses in mice (88). Submucosal DCs migrating to the draining lymph node have been shown to induce Th1 responses to genital HSV-2 infection in mice (89). Furthermore, both monocyte-derived DCs and submucosal lamina propria DCs stimulate the protective responses of memory CD8+ T cells established by HSV-2 333 TK- immunization in mice $(33,50)$. Thus, type I IFNs promote both local and systemic DC-facilitated Th1 immune responses during HSV-2 infection.

Similarly, IFN- $\gamma$ plays a critical role in stimulating APC functions for the development of adaptive immunity. Activation of macrophages and upregulation of epithelial MHC class II expression by IFN- $\gamma$ signaling contributes to the development of effective $\mathrm{T}$ cell immunity $(57,58)$. Similarly, the kinetic differences in IFN- $\gamma$ production between genital HSV-1 and HSV-2 infection in mice define their pathological outcomes, as early NK cell-derived IFN- $\gamma$ production following genital HSV-1 infection induced rapid DC maturation and migration to the draining lymph node to protect against neuroinvasion (90). However, genital HSV-2 infection in mice did not elicit the same early burst of IFN- $\gamma$ production and resulted in greater nervous system infection and more severe disease (90). IFN- $\gamma$ signaling further promotes $\mathrm{T}$ cell responses by regulating the expression of costimulatory molecule $\mathrm{B} 7$ 
isoforms on APCs (26). However, IFN- $\gamma$ treatment of human PBMCs failed to enhance B7-1 or B7-2 expression on monocytes from individuals with recurrent HSV-2 infection, which may explain impaired $\mathrm{T}$ cell-mediated immunity and viral clearance in these individuals (26). Overall, these studies demonstrate how inducing a potent and early IFN- $\gamma$ response is critical in APC function and mounting an effective and protective adaptive immune response to HSV-2.

\section{T Cells}

$\mathrm{CD}^{+} \mathrm{T}$ cells provide fundamental antiviral protection by perforin and granzyme-mediated lysis of infected cells as well as production of the type II IFN, IFN- $\boldsymbol{\gamma}(31)$. Additionally, IFN- $\boldsymbol{\gamma}$ production by $\mathrm{T}$ cells is an essential component of the memory response to HSV2 reinfection, as HSV-2 $333 \mathrm{TK}^{-}$immunized $\mathrm{CD}^{-/-}$mice cannot clear genital HSV-2 infections and do not survive lethal HSV-2 challenge $(32,91)$. This protection during reinfection is mediated by $\mathrm{CD} 4{ }^{+} \mathrm{T}$ cell-derived IFN- $\gamma$ as well as their ability to orchestrate enhanced NK cell activation and IFN- $\gamma$ production (92). Further vaccination studies eliciting tissue resident memory $\mathrm{CD}^{+} \mathrm{T}$ cells suggest IFN- $\gamma$ is required for vaccination-induced protection against HSV-2 (33).

Type I IFNs promote $\mathrm{T}$ cell effector function indirectly via APCs, as described in section 4.1. Additionally, type I IFNs have been shown to directly act on mouse $\mathrm{T}$ cells to promote expansion, memory formation, and effector functions that are critical for HSV-2 clearance (93-96). However, type I IFNs may also possess immunoregulatory functions to prevent excessive $\mathrm{T}$ cell activation and subsequent tissue damage. High levels of type I IFN signaling through Poly(I:C) and IFN- $\alpha / \beta$ treatment in vivo has been seen to induce attrition or reduced proliferation of $\mathrm{CD}^{+} \mathrm{T}$ cells (97-99). Thus, an appropriate type I IFN response is critical to balance activation and regulation of $\mathrm{T}$ cell responses during HSV-2 infection.

As we have previously outlined, type I IFNs critically regulate NK cell and T cell-derived production of IFN- $\gamma$. The development of effective T cell responses to HSV-2 infection is also dependent on IFN- $\gamma$. IFN- $\gamma$ stimulates chemokine production for the recruitment of immune cells, as mouse HSV-2 vaccination has been associated with induction of local IFN- $\gamma$-dependent RANTES production (100). Similarly, HSV-2 vaccination in mice can also facilitate retention of vaginal tissue resident memory $\mathrm{CD}^{+} \mathrm{T}$ cells by IFN- $\gamma$-dependent macrophage production of CCL5 and CXCL9 (101). As seen above, IFN- $\gamma$ promotes APC maturation and facilitation of Th1 responses to HSV-2 infection. In contrast, dysregulation or deficiency of IFN- $\gamma$ in HSV-2-infected individuals and mice has been observed to promote a Th2 cytokine response through IL-10 in humans and IL-4 in mice, which is unable to facilitate viral clearance $(26,91)$. Th2 cytokine driven immune responses to vaginal HSV-2 infection in mice have also been described as pathogenic (102). Thus, type II IFNs are critical mediators of $\mathrm{T}$ cell-mediated viral clearance, and prevent pathogenic Th2 immunity during primary and secondary HSV-2 infection. An effective vaccine against HSV-2 must elicit potent T cell-mediated IFN- $\gamma$ release for optimal protection against HSV-2 infection.

\section{B Cells}

A role for B cell-mediated innate protection against HSV-2 infection has been described, as B cell-deficient mice exhibited transient infection and inflammation, while $\mathrm{T}$ cell-deficient mice did not exhibit infection-induced inflammation (32). Passive transfer of serum from uninfected mice reduced vaginal HSV-2 titers, suggesting innate protection by natural antibodies. Thus, the sheer presence of antibodies in the vaginal mucosa contributes to innate-like protection against HSV-2 infection. Further, naïve B cells and antibody-secreting cells have recently been identified in recurrent HSV-2 lesions alongside $\mathrm{CD}^{+} \mathrm{T}$ cells, suggesting a role for B cells in the resolution of reactivated HSV-2 lesions (103).

$\mathrm{B}$ cells also contribute to protective memory responses against HSV-2 reinfection following immunization with HSV-2 $333 \mathrm{TK}^{-}$ and live-attenuated $0 \Delta$ NLS strains of HSV-2 (104, 105). Several studies have shown that type I IFNs directly and indirectly regulate $\mathrm{B}$ cell proliferation, plasma cell differentiation, and isotype switching (93, 106-109). Thus, type I IFN signaling promotes induction of memory B cell development and vaccine efficacy. Likewise, type II IFN responses are fundamental for B cell recruitment, priming, and function during primary and secondary HSV-2 infection. Upon secondary challenge, IFN- $\gamma$ was shown to mediate the production of chemokines, such as CXCL9 and CXCL10, that recruit memory B cells that are capable of rapid antibody secretion (110). CD4 ${ }^{+} \mathrm{T}$ cell IFN $-\gamma$ production following immunization also plays an important role in antibody access to neuronal tissues to enable viral control (111). In another study, IFN- $\gamma$ deficiency did not impact overall serum IgG levels in $\mathrm{TK}^{-}$immunized mice (91). Instead, they found that IgG1 antibodies were enriched in IFN- $\gamma^{-/-}$mice, whereas IgG2 was favoured in WT mice. Although this did not significantly influence early protection against HSV-2, these IgG1 antibodies were less effective in preventing HSV-2 spread to the nervous system (91). This suggests that dysregulation of IFN- $\gamma$, as observed in individuals with recurrent HSV-2 infection $(26,28)$, may support viral entry into the CNS and the establishment of latent infection via altered isotype switching. Thus, IFN- $\gamma$ is critically required to recruit and promote optimal $\mathrm{B}$ cell responses, while preventing suboptimal isotype switching, to protect against HSV-2 infection.

\section{The Role of IFNs in HSV-2 Vaccine Development}

Mouse models of HSV-2 vaccination have largely demonstrated potent memory $\mathrm{T}$ cell and neutralizing antibody responses capable of protection following subsequent HSV-2 challenge (29, 32, 33, 91, $92,100,111)$. However, these preclinical results have not translated to vaccine efficacy in humans during clinical trials. Several candidate vaccines have exhibited poor efficacy in clinical trials with varying induction of neutralizing antibody or cellular responses (112-117). Although vaccination models in mice demonstrate the requirement for memory $\mathrm{CD}^{+} \mathrm{T}$ cell-derived IFN- $\gamma$ for optimal protection, most clinical studies in humans focus on neutralizing antibody titer and provide little to no examination of $\mathrm{CD}^{+} \mathrm{T}$ cell specificity or IFN- $\gamma$ production $(113,116-118)$.

Although type I IFNs may not be required for vaccineinduced memory responses in mice (119), HSV-2 evasion of 
type I IFN signaling, demonstrated by a lack of type I IFN in human lesion biopsies compared to mice, may reduce the efficacy of vaccine-induced memory responses (120). As evidenced by multiple clinical trial failures despite successful pre-clinical models, the factors behind the development of protective adaptive immunity to genital HSV-2 infection differ between human and animal models. In addition, vaccination studies in mice do not consider the role of type I IFN in regard to the longevity of the established protective immunity, with studies administering secondary challenge under a month post-vaccination (119). The role of type III IFNs should also be carefully considered, as the use of IFN- $\lambda 3$ as an adjuvant in a mouse vaccination model enhanced both humoral and cellular immune responses, resulting in improved vaccine efficacy (121). Thus, a lack of type I, II, and III IFN induction by HSV-2 vaccination in humans may prevent the development of protective adaptive immunity and should be carefully considered in future vaccine development.

\section{CONCLUDING REMARKS}

The development of novel preventative and prophylactic treatments to sexually transmitted HSV-2 has been hindered by an inability to induce a strong, protective immune response in the genital mucosa. As summarized in Figure 1, the role of IFNs extends beyond suppressing viral replication, but also in the development of local protective innate and adaptive immune responses to viral infection. Type I and II IFN signaling directly, and indirectly through the induction of innate immunity, regulates the development of immune memory and adaptive responses to viral infection. Similarly, type III IFNs also play a

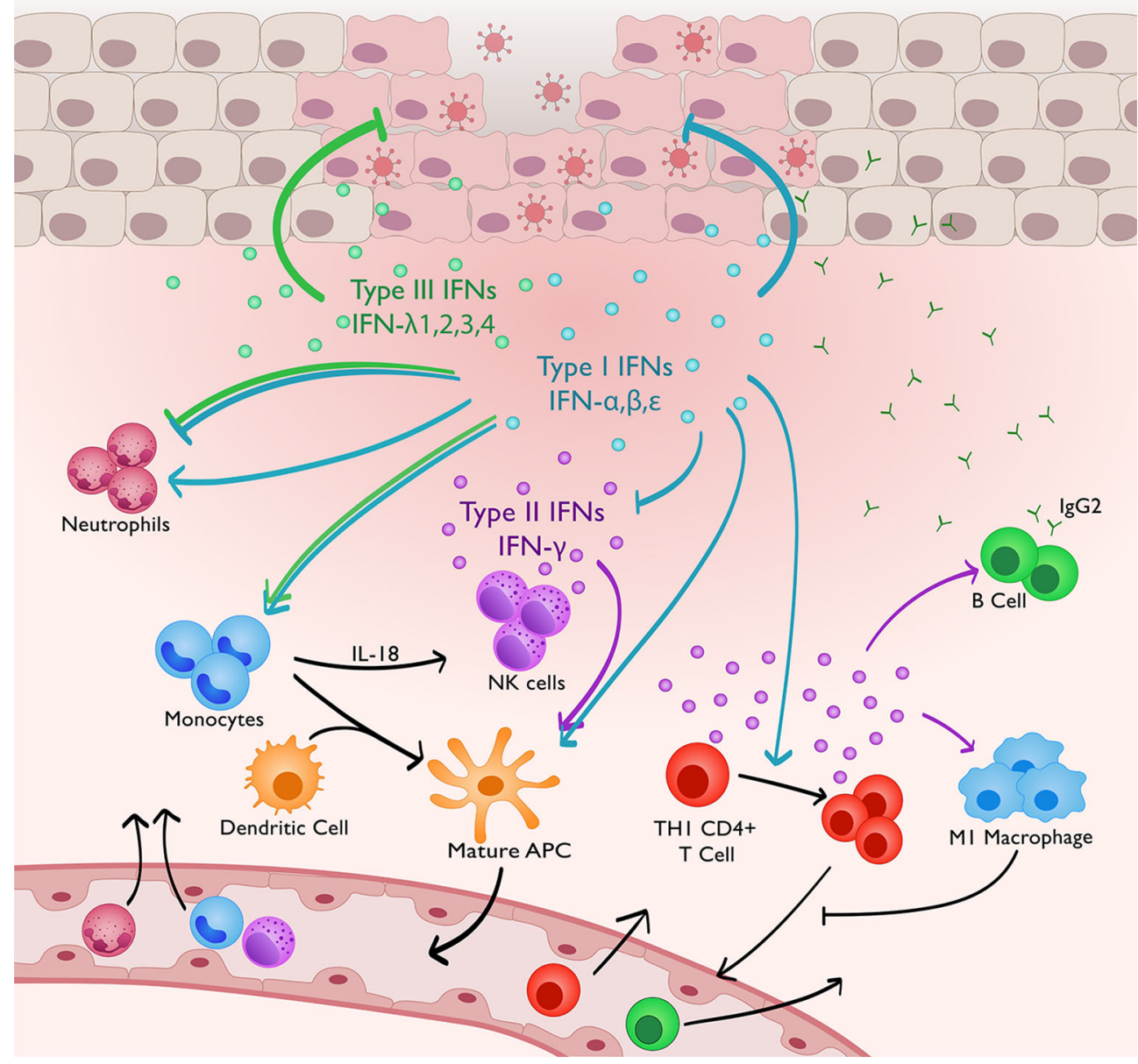

FIGURE 1 | IFNs regulating innate and adaptive immunity to genital HSV-2 infection. Type I and III IFNs directly suppress HSV-2 replication in the genital mucosa. Concurrently, type I IFN signaling promotes IM recruitment and production of IL-18. This in turns stimulates NK cell IFN- $\gamma$ production. Type I IFNs stimulate adaptive immunity both directly and indirectly through IM differentiation into APCs, maturation of DCs, and inducing CD4+ T cell expansion. Type I IFNs can both promote neutrophil function, and negatively regulate innate immune responses to prevent immune-mediated pathology by suppressing neutrophil recruitment and limiting IFN- $\gamma$ production by NK cells. IFN- $\gamma$ production, highly dependent on type I IFN signaling, promotes IgG2 antibody production, M1 macrophage polarisation and DC maturation, which will promote CD4+ memory T cell retention via production of CCL4 and CXCL9. Type III IFNs, similar to type I IFNs, have also been shown to suppress neutrophil activity and monocyte-mediated NK cell activation, but this is not yet demonstrated in the vaginal mucosa. 
critical role in controlling viral replication, and likely have many additional unexplored functions in regulating innate immune responses in the genital mucosa. Impaired type I IFN signaling during genital herpes infection will consequently impair type II IFN responses, which aid in the development of adaptive immunity and are critical for HSV-2 clearance. As previously described, dysregulated responses to IFN- $\gamma$ are observed in recurrent HSV-2-infected individuals (26). Future treatments or vaccines must restore responsiveness to IFN- $\gamma$ signaling and elicit potent $\mathrm{CD}^{+}{ }^{+} \mathrm{T}$ cell-derived IFN- $\gamma$ production due to its multifaceted role in immune regulation and antiviral immunity.

As exemplified by this review, proper induction of type I, II, and III IFNs is critical for the development of immunity and protective memory against genital HSV-2 infections. The use of IFNs as a therapeutic strategy against HSV-2 could provide a promising avenue. In addition, a greater research focus on the role of type III IFNs should be placed, including both their immunoregulatory functions and their potential as a treatment against shedding and reactivation. While type I IFNs offer invaluable protection during the early stages of infection, their presence can also promote pathogenic immune responses during mucosal infection $(67,122)$. Type III IFN treatment of various other viral infections, such as with SARS-CoV-2 patients and an

\section{REFERENCES}

1. James C, Harfouche M, Welton NJ, Turner KME, Abu-Raddad LJ, Gottlieb SL, et al. Herpes Simplex Virus: Global Infection Prevalence and Incidence Estimates, 2016. Bull World Health Organ (2020) 98:315-29. doi: 10.2471/ BLT.19.237149

2. Jiang YC, Feng H, Lin YC, Guo XR. New Strategies Against Drug Resistance to Herpes Simplex Virus. Int J Oral Sci (2016) 8:1-6. doi: 10.1038/ijos.2016.3

3. Schiffer JT, Corey L. Rapid Host Immune Response and Viral Dynamics in Herpes Simplex Virus-2 Infection. Nat Med (2013) 19:280-90. doi: 10.1038/ nm.3103

4. Kimberlin DW, Baley J. Guidance on Management of Asymptomatic Neonates Born to Women With Active Genital Herpes Lesions. Pediatrics (2013) 131:e635-46. doi: 10.1542/peds.2012-3216

5. Brown ZA, Gardella C, Wald A, Morrow RA, Corey L. Genital Herpes Complicating Pregnancy. Obstet Gynecol (2005) 106:845-56. doi: 10.1097/ 01.AOG.0000180779.35572.3a

6. Tronstein E, Johnston C, Huang ML, Selke S, Magaret A, Warren T, et al. Genital Shedding of Herpes Simplex Virus Among Symptomatic and Asymptomatic Persons With HSV-2 Infection. JAMA (2011) 305:1441-9. doi: 10.1001/jama.2011.420

7. Wald A, Zeh J, Selke S, Warren T, Ryncarz AJ, Ashley R, et al. Reactivation of Genital Herpes Simplex Virus Type 2 Infection in Asymptomatic Seropositive Persons. N Engl J Med (2000) 342:844-50. doi: 10.1056/ NEJM200003233421203

8. Koelle DM, Wald A. Herpes Simplex Virus: The Importance of Asymptomatic Shedding. J Antimicrob Chemother (2000) 45:1-8. doi: 10.1093/jac/45.suppl_4.1

9. Johnston C, Saracino M, Kuntz S, Magaret A, Selke S, Huang M, et al. Standard-Dose and High-Dose Daily Antiviral Therapy for Short Episodes of Genital HSV-2 Reactivation: Three Randomised, Open-Label, Cross-Over Trials. Lancet (2012) 379:641-7. doi: 10.1016/S0140-6736(11)61750-9

10. Van de Perre P, Segondy M, Foulongne V, Ouedraogo A, Konate I, Huraux JM, et al. Herpes Simplex Virus and HIV-1: Deciphering Viral Synergy. Lancet Infect Dis (2008) 8:490-7. doi: 10.1016/S1473-3099(08)70181-6

11. Looker KJ, Elmes JAR, Gottlieb SL, Schiffer JT, Vickerman P, Turner KME, et al. Effect of HSV-2 Infection on Subsequent HIV Acquisition: An Updated
IAV mouse model, have demonstrated it to be both safe and effective $(123,124)$. Overall, both the antiviral and regulatory functions of IFNs in vaginal HSV-2 infection must be carefully and seriously considered in the development of novel therapeutics, prophylactic treatments, and vaccines.

\section{AUTHOR CONTRIBUTIONS}

$\mathrm{EF}$ and $\mathrm{EB}$ conceptualised the review. $\mathrm{EF}, \mathrm{EB}$, and $\mathrm{FV}$ were responsible for writing the manuscript. EF, EB and AA edited the manuscript. AA supervised and guided the development of the manuscript. All authors contributed to the article and approved the submitted version.

\section{FUNDING}

This work was supported by the Canadian Institutes of Health Research (CIHR). AA is also a recipient of a CIHR Tier 1 Canada Research Chair. EF is supported by the Ontario Graduate Scholarship. EB is a recipient of the CIHR Canada Graduate Scholarship-Master's.

Systematic Review and Meta-Analysis. Lancet Infect Dis (2017) 17:1303-16 doi: 10.1016/S1473-3099(17)30405-X

12. Tognarelli EI, Palomino TF, Corrales N, Bueno SM, Kalergis AM, González PA. Herpes Simplex Virus Evasion of Early Host Antiviral Responses. Front Cell Infect Microbiol (2019) 9:127. doi: 10.3389/fcimb.2019.00127

13. Platanias LC. Mechanisms of Type-I- and Type-II- Mediated Signalling. Nat Rev Immunol (2005) 5:375-86. doi: 10.1038/nri1604

14. Gill N, Deacon PM, Lichty B, Mossman KL, Ashkar AA, Al GET. Induction of Innate Immunity Against Herpes Simplex Virus Type 2 Infection via Local Delivery of Toll-Like Receptor Ligands Correlates With Beta Interferon Production. J Virol (2006) 80:9943-50. doi: 10.1128/ JVI.01036-06

15. Conrady CD, Halford WP, Carr DJJ. Loss of the Type I Interferon Pathway Increases Vulnerability of Mice to Genital Herpes Simplex Virus 2 Infection. J Virol (2011) 85:1625-33. doi: 10.1128/JVI.01715-10

16. Lund J, Sato A, Akira S, Medzhitov R, Iwasaki A. Toll-Like Receptor 9Mediated Recognition of Herpes Simplex Virus-2 by Plasmacytoid Dendritic Cells. J Exp Med (2003) 198:513-20. doi: 10.1084/jem.20030162

17. Melchjorsen J, Rintahaka J, Søby S, Horan KA, Poltajainen A, Østergaard L, et al. Early Innate Recognition of Herpes Simplex Virus in Human Primary Macrophages Is Mediated via the MDA5/MAVS-Dependent and MDA5/ MAVS/RNA Polymerase III-Independent Pathways. J Virol (2010) 84:11350-8. doi: 10.1128/jvi.01106-10

18. Eriksson K, Svensson A, Hait AS, Schlüter K, Tunbäck P, Nordström I, et al. Cutting Edge: Genetic Association Between IFI16 Single Nucleotide Polymorphisms and Resistance to Genital Herpes Correlates With IFI16 Expression Levels and HSV-2-Induced IFN- $\beta$ Expression. J Immunol (2017) 199:2613-7.

19. Skouboe MK, Knudsen A, Reinert LS, Boularan C, Lioux T, Perouzel E, et al STING Agonists Enable Antiviral Cross-Talk Between Human Cells and Confer Protection Against Genital Herpes in Mice. PloS Pathog (2018) 14: e1005256. doi: 10.1371/journal.ppat.1006976

20. Fung KY, Mangan NE, Cumming H, Horvat JC, Mayall JR, Stifter SA, et al. Interferon- Protects the Female Reproductive Tract From Viral and Bacterial Infection. Sci (80- ) (2013) 339:1088-92. doi: 10.1126/science.1233321

21. Lund JM, Linehan MM, Iijima N, Iwasaki A. Cutting Edge: Plasmacytoid Dendritic Cells Provide Innate Immune Protection Against Mucosal Viral 
Infection In Situ. J Immunol (2006) 177:7510-4. doi: 10.4049/jimmunol. 177.11.7510

22. Rasmussen SB, Sørensen LN, Malmgaard L, Ank N, Baines JD, Chen ZJ, et al. Type I Interferon Production During Herpes Simplex Virus Infection Is Controlled by Cell-Type-Specific Viral Recognition Through Toll-Like Receptor 9, the Mitochondrial Antiviral Signaling Protein Pathway, and Novel Recognition Systems. J Virol (2007) 81:13315-24. doi: 10.1128/jvi.01167-07

23. Donaghy H, Bosnjak L, Harman AN, Marsden V, Tyring SK, Meng T-C, et al. Role for Plasmacytoid Dendritic Cells in the Immune Control of Recurrent Human Herpes Simplex Virus Infection. J Virol (2009) 83:195261. doi: 10.1128/jvi.01578-08

24. Swiecki M, Wang Y, Gilfillan S, Colonna M. Plasmacytoid Dendritic Cells Contribute to Systemic But Not Local Antiviral Responses to HSV Infections. PloS Pathog (2013) 9:e1003728. doi: 10.1371/journal.ppat.1003728

25. Lee AJ, Chen B, Chew MV, Barra NG, Shenouda MM, Nham T, et al. Inflammatory Monocytes Require Type I Interferon Receptor Signaling to Activate NK Cells via IL-18 During a Mucosal Viral Infection. J Exp Med (2017) 214:1153-67. doi: 10.1084/jem.20160880

26. Singh R, Kumar A, Creery WD, Ruben M, Giulivi A, Diaz-Mitoma F. Dysregulated Expression of IFN- $\gamma$ and IL-10 and Impaired IFN- $\gamma$-Mediated Responses at Different Disease Stages in Patients With Genital Herpes Simplex Virus-2 Infection. Clin Exp Immunol (2003) 133:97-107. doi: 10.1046/j.1365-2249.2003.02183.x

27. Carmack MA, Yasukawa LL, Chang SY, Tran C, Saldana F, Arvin AM, et al. $\mathrm{T}$ Cell Recognition and Cytokine Production Elicited by Common and Type- Specific Glycoproteins of Herpes Simplex Virus Type 1 and Type 2. J Infect Dis (1996) 174:899-906. doi: 10.1093/infdis/174.5.899

28. Svensson A, Tunback P, Nordstrom I, Shestakov A, Padyukov L, Eriksson K. STAT4 Regulates Antiviral Gamma Interferon Responses and Recurrent Disease During Herpes Simplex Virus 2 Infection. J Virol (2012) 86:9409-15. doi: 10.1128/jvi.00947-12

29. Milligan GN, Bernstein DI, Bourne N. T Lymphocytes are Required for Protection of the Vaginal Mucosae and Sensory Ganglia of Immune Mice Against Reinfection With Herpes Simplex Virus Type 2. J Immunol (1998) 160:6093-100. doi: 10.4049/jimmunol.160126100

30. Milligan GN, Dudley-McClain KL, Young CG, Chu CF. T-Cell-Mediated Mechanisms Involved in Resolution of Genital Herpes Simplex Virus Type 2 (HSV-2) Infection of Mice. J Reprod Immunol (2004) 61:115-27. doi: 10.1016/j.jri.2003.12.002

31. Dobbs ME, Strasser JE, Chu C-F, Chalk C, Milligan GN. Clearance of Herpes Simplex Virus Type 2 by CD8+ T Cells Requires Gamma Interferon and Either Perforin- or Fas-Mediated Cytolytic Mechanisms. J Virol (2005) 79:14546-54. doi: 10.1128/jvi.79.23.14546-14554.2005

32. Harandi AM, Svennerholm B, Holmgren J, Eriksson K. Differential Roles of B Cells and IFN- $\gamma$-Secreting CD4+ T Cells in Innate and Adaptive Immune Control of Genital Herpes Simplex Virus Type 2 Infection in Mice. J Gen Virol (2001) 82:845-53. doi: 10.1099/0022-1317-82-4-845

33. Shin H, Kumamoto Y, Gopinath S, Iwasaki A. CD301b+ Dendritic Cells Stimulate Tissue-Resident Memory CD8+ T Cells to Protect Against Genital HSV-2. Nat Commun (2016) 7:1-10. doi: 10.1038/ncomms13346

34. Lazear HM, Nice TJ, Diamond MS. Interferon- $\lambda$ : Immune Functions at Barrier Surfaces and Beyond. Immunity (2015) 43:15-28. doi: 10.1016/ j.immuni.2015.07.001

35. Kotenko SV, Durbin JE. Contribution of Type III Interferons to Antiviral Immunity: Location, Location, Location. J Biol Chem (2017) 292:7295-303. doi: $10.1074 /$ jbc.R117.777102

36. Iversen MB, Ank N, Melchjorsen J, Paludan SR. Expression of Type III Interferon (IFN) in the Vaginal Mucosa Is Mediated Primarily by Dendritic Cells and Displays Stronger Dependence on NF-kb Than Type I IFNs. J Virol (2010) 84:4579-86. doi: 10.1128/jvi.02591-09

37. Coccia EM, Severa M, Giacomini E, Monneron D, Remoli ME, Julkunen I, et al. Viral Infection and Toll-Like Receptor Agonists Induce a Differential Expression of Type I and $\lambda$ Interferons in Humans Plasmacytoid and Monocyte-Derived Dendritic Cells. Eur J Immunol (2004) 34:796-805. doi: 10.1002/eji.200324610

38. Yin Z, Dai J, Deng J, Sheikh F, Natalia M, Shih T, et al. Type III IFNs Are Produced by and Stimulate Human Plasmacytoid Dendritic Cells. J Immunol (2012) 189:2735-45. doi: 10.4049/jimmunol.1102038
39. Zhou Z, Hamming OJ, Ank N, Paludan SR, Nielsen AL, Hartmann R. Type III Interferon (IFN) Induces a Type I IFN-Like Response in a Restricted Subset of Cells Through Signaling Pathways Involving Both the Jak-STAT Pathway and the Mitogen-Activated Protein Kinases. J Virol (2007) 81:774958. doi: $10.1128 /$ jvi.02438-06

40. Sheppard P, Kindsvogel W, Xu W, Henderson K, Schlutsmeyer S, Whitmore TE, et al. IL-28, IL-29 and Their Class II Cytokine Receptor IL-28r. Nat Immunol (2003) 4:63-8. doi: 10.1038/ni873

41. Sommereyns C, Paul S, Staeheli P, Michiels T. IFN-Lambda (IFN- $\lambda$ ) is Expressed in a Tissue-Dependent Fashion and Primarily Acts on Epithelial Cells In Vivo. PloS Pathog (2008) 4:e1003728. doi: 10.1371/journal. ppat.1000017

42. Kotenko SV, Gallagher G, Baurin VV, Lewis-Antes A, Shen M, Shah NK, et al. IFN- $\lambda$ s Mediate Antiviral Protection Through a Distinct Class II Cytokine Receptor Complex. Nat Immunol (2003) 4:69-77. doi: 10.1038/ni875

43. Ank N, West H, Bartholdy C, Eriksson K, Thomsen AR, Paludan SR. Lambda Interferon (IFN- ), a Type III IFN, Is Induced by Viruses and IFNs and Displays Potent Antiviral Activity Against Select Virus Infections In Vivo. J Virol (2006) 80:4501-9. doi: 10.1128/jvi.80.9.4501-4509.2006

44. Ank N, Iversen MB, Bartholdy C, Staeheli P, Hartmann R, Jensen UB, et al. An Important Role for Type III Interferon (IFN- $\lambda /$ IL-28) in TLR-Induced Antiviral Activity. J Immunol (2008) 180:2474-85. doi: 10.4049/jimmunol. 180.4.2474

45. Gillgrass AE, Fernandez SA, Rosenthal KL, Kaushic C. Estradiol Regulates Susceptibility Following Primary Exposure to Genital Herpes Simplex Virus Type 2, While Progesterone Induces Inflammation. J Virol (2005) 79:310716. doi: $10.1128 /$ JVI.79.5.3107

46. Seillet C, Laffont S, Trémollières F, Rouquié N, Ribot C, Arnal JF, et al. The TLR-Mediated Response of Plasmacytoid Dendritic Cells Is Positively Regulated by Estradiol In Vivo Through Cell-Intrinsic Estrogen Receptor $\alpha$ Signaling. Blood (2012) 119:454-64. doi: 10.1182/blood-2011-08-371831

47. Hughes GC, Thomas S, Li C, Kaja M-K, Clark EA. Cutting Edge: Progesterone Regulates IFN- $\alpha$ Production by Plasmacytoid Dendritic Cells. J Immunol (2008) 180:2029-33. doi: 10.4049/jimmunol.180.4.2029

48. Kaushic C, Ashkar AA, Reid LA, Rosenthal KL. Progesterone Increases Susceptibility and Decreases Immune Responses to Genital Herpes Infection. J Virol (2003) 77:4558-65. doi: 10.1128/jvi.77.8.4558-4565.2003

49. Gillgrass AE, Ashkar AA, Rosenthal KL, Kaushic C. Prolonged Exposure to Progesterone Prevents Induction of Protective Mucosal Responses Following Intravaginal Immunization With Attenuated Herpes Simplex Virus Type 2. J Virol (2003) 77:9845-51. doi: 10.1128/JVI.77.18.9845

50. Iijima N, Mattei LM, Iwasaki A. Recruited Inflammatory Monocytes Stimulate Antiviral Th1 Immunity in Infected Tissue. Proc Natl Acad Sci USA (2011) 108:284-9. doi: 10.1073/pnas.1005201108

51. Krzyzowska M, Baska P, Orlowski P, Zdanowski R, Winnicka A, Eriksson K, et al. HSV-2 Regulates Monocyte Inflammatory Response via the Fas/FasL Pathway. PloS One (2013) 8:e70308. doi: 10.1371/journal.pone.0070308

52. Krzyzowska M, Shestakov A A, Eriksson K K, F Chiodi F. Role of Fas/FasL in Regulation of Inflammation in Vaginal Tissue During HSV-2 Infection. Cell Death Dis (2011) 2:1-12. doi: 10.1038/cddis.2011.14

53. Fujikura D, Chiba S, Muramatsu D, Kazumata M, Nakayama Y, Kawai T, et al. Type-I Interferon is Critical for FasL Expression on Lung Cells to Determine the Severity of Influenza. PloS One (2013) 8:e55321. doi: 10.1371/ journal.pone.0055321

54. Uyangaa E, Kim JH, Patil AM, Choi JY, Kim SB, Eo SK. Distinct Upstream Role of Type I IFN Signaling in Hematopoietic Stem Cell-Derived and Epithelial Resident Cells for Concerted Recruitment of Ly-6chi Monocytes and NK Cells via CCL2-CCL3 Cascade. PloS Pathog (2015) 11:e1005256. doi: 10.1371 /journal.ppat.1005256

55. Conrady CD, Zheng M, Mandal NA, Van Rooijen N, Carr DJJ. IFN$\alpha$-Driven CCL2 Production Recruits Inflammatory Monocytes to Infection Site in Mice. Mucosal Immunol (2013) 6:45-55. doi: 10.1038/ mi.2012.46

56. Baskin H, Ellermann-Eriksen S, Lovmand J, Mogensen SC. Herpes Simplex Virus Type 2 Synergizes With Interferon- $\gamma$ in the Induction of Nitric Oxide Production in Mouse Macrophages Through Autocrine Secretion of Tumour Necrosis Factor- $\alpha$. J Gen Virol (1997) 78:195-203. doi: 10.1099/ 0022-1317-78-1-195 
57. Lee AJ, Ashkar AA. The Dual Nature of Type I and Type II Interferons. Front Immunol (2018) 9:2061. doi: 10.3389/fimmu.2018.02061

58. Parr MB, Parr EL. The Role of Gamma Interferon in Immune Resistance to Vaginal Infection by Herpes Simplex Virus Type 2 in Mice. Virology (1999) 294:282-94. doi: 10.1006/viro.1999.9739

59. Milligan GN. Neutrophils Aid in Protection of the Vaginal Mucosae of Immune Mice Against Challenge With Herpes Simplex Virus Type 2. J Virol (1999) 73:6380-6. doi: 10.1128/jvi.73.8.6380-6386.1999

60. Gardner JK, Swaims-Kohlmeier A, Herbst-Kralovetz MM. IL-36 $\gamma$ Is a Key Regulator of Neutrophil Infiltration in the Vaginal Microenvironment and Limits Neuroinvasion in Genital HSV-2 Infection. J Immunol (2019) 203:2655-64. doi: 10.4049/jimmunol.1900280

61. Wang P, Gamero AM, Jensen LE. IL-36 Promotes Anti-Viral Immunity by Boosting Sensitivity to IFN- $\alpha / \beta$ in IRF1 Dependent and Independent Manners. Nat Commun (2019) 10:4700. doi: 10.1038/s41467-019-12318-y

62. Seo SU, Kwon HJ, Ko HJ, Byun YH, Seong BL, Uematsu S, et al. Type I Interferon Signaling Regulates Ly6Chi Monocytes and Neutrophils During Acute Viral Pneumonia in Mice. PloS Pathog (2011) 7:e1001304. doi: 10.1371/journal.ppat.1001304

63. Kulkarni U, Zemans RL, Smith CA, Wood SC, Deng JC, Goldstein DR. Excessive Neutrophil Levels in the Lung Underlie the Age-Associated Increase in Influenza Mortality. Mucosal Immunol (2019) 12:545-54. doi: 10.1038/s41385-018-0115-3

64. Stout-Delgado HW, Du W, Shirali AC, Booth CJ, Goldstein DR. Aging Promotes Neutrophil-Induced Mortality by Augmenting IL-17 Production During Viral Infection. Cell Host Microbe (2009) 6:446-56. doi: 10.1016/ j.chom.2009.09.011

65. Stock AT, Smith JM, Carbone FR. Type I IFN Suppresses Cxcr2 Driven Neutrophil Recruitment Into the Sensory Ganglia During Viral Infection. J Exp Med (2014) 211:751-9. doi: 10.1084/jem.20132183

66. Broggi A, Tan Y, Granucci F, Zanoni I. IFN- $\lambda$ Suppresses Intestinal Inflammation by non-Translational Regulation of Neutrophil Function. Nat Immunol (2017) 18:1084-93. doi: 10.1038/ni.3821

67. Lebratti T, Lim YS, Cofie A, Andhey P, Jiang X, Scott J, et al. A Sustained Type I IFN-Neutrophil-IL-18 Axis Drives Pathology During Mucosal Viral Infection. Elife (2021) 10:e65762. doi: 10.7554/eLife.65762

68. Orange JS. Human Natural Killer Cell Deficiencies and Susceptibility to Infection. Microbes Infect (2002) 4:1545-58. doi: 10.1016/S1286-4579(02) 00038-2

69. Ashkar AA, Rosenthal KL. Interleukin-15 and Natural Killer and NKT Cells Play a Critical Role in Innate Protection Against Genital Herpes Simplex Virus Type 2 Infection. J Virol (2003) 77:10168-71. doi: 10.1128/ JVI.77.18.10168

70. Thapa M, Welner RS, Pelayo R, Carr DJJ. CXCL9 and CXCL10 Expression Are Critical for Control of Genital Herpes Simplex Virus Type 2 Infection Through Mobilization of HSV-Specific CTL and NK Cells to the Nervous System. J Immunol (2008) 180:1098-106. doi: 10.4049/jimmunol.180.2.1098

71. Thapa M, Kuziel WA, Carr DJJ. Susceptibility of CCR5-Deficient Mice to Genital Herpes Simplex Virus Type 2 Is Linked to NK Cell Mobilization. J Virol (2007) 81:3704-13. doi: 10.1128/jvi.02626-06

72. Gill N, Chenoweth MJ, Verdu EF, Ashkar AA. NK Cells Require Type I IFN Receptor for Antiviral Responses During Genital HSV-2 Infection. Cell Immunol (2011) 269:29-37. doi: 10.1016/j.cellimm.2011.03.007

73. Martinez J, Huang X, Yang Y, Alerts E. Direct Action of Type I IFN on NK Cells Is Required for Their Activation in Response to Vaccinia Viral Infection In Vivo. J Immunol (2008) 180:1592-87. doi: 10.4049/jimmunol.180.3.1592

74. Swann JB, Hayakawa Y, Zerafa N, Sheehan KCF, Scott B, Schreiber RD, et al. Type I IFN Contributes to NK Cell Homeostasis, Activation, and Antitumor Function. J Immunol (2007) 178:7540-9. doi: 10.4049/jimmunol.178.12.7540

75. Mack EA, Kallal LE, Demers DA, Biron CA. Type 1 Interferon Induction of Natural Killer Cell Gamma Interferon Production for Defense During Lymphocytic Choriomeningitis Virus Infection. MBio (2011) 2:13-5. doi: 10.1128/mBio.00169-11

76. Madera S, Rapp M, Firth MA, Beilke JN, Lanier LL, Sun JC. Type I IFN Promotes NK Cell Expansion During Viral Infection by Protecting NK Cells Against Fratricide. J Exp Med (2016) 213:225-33. doi: 10.1084/jem.20150712

77. Sun JC, Madera S, Bezman NA, Beilke JN, Kaplan MH, Lanier LL. Proinflammatory Cytokine Signaling Required for the Generation of
Natural Killer Cell Memory. J Exp Med (2012) 209:947-54. doi: 10.1084/ jem. 20111760

78. Abdul-Careem MF, Lee AJ, Pek EA, Gill N, Gillgrass AE, Chew MV, et al. Genital HSV-2 Infection Induces Short-Term NK Cell Memory. PloS One (2012) 7:e32821. doi: 10.1371/journal.pone.0032821

79. Lucas M, Schachterle W, Oberle K, Aichele P, Diefenbach A. Dendritic Cells Prime Natural Killer Cells by Trans-Presenting Interleukin 15. Immunity (2007) 26:503-17. doi: 10.1016/j.immuni.2007.03.006

80. Wang Y, Li T, Chen Y, Wei H, Sun R, Tian Z. Involvement of NK Cells in IL28b-Mediated Immunity Against Influenza Virus Infection. J Immunol (2017) 199:1012-20. doi: 10.4049/jimmunol.1601430

81. Lauwerys BR, Garot N, Renauld J-C, Houssiau FA. Cytokine Production and Killer Activity of NK/T-NK Cells Derived With IL-2, IL-15, or the Combination of IL-12 and IL-18. J Immunol (2000) 165:1847-53. doi: 10.4049/jimmunol.165.4.1847

82. Chaix J, Tessmer MS, Hoebe K, Fuséri N, Ryffel B, Dalod M, et al. Cutting Edge: Priming of NK Cells by IL-18. J Immunol (2008) 181:1627-31. doi: 10.4049/jimmunol.181.3.1627

83. Poznanski SM, Lee AJ, Nham T, Lusty E, Larché MJ, Lee DA, et al. Combined Stimulation With Interleukin-18 and Interleukin-12 Potently Induces Interleukin-8 Production by Natural Killer Cells. J Innate Immun (2017) 9:511-25. doi: 10.1159/000477172

84. Lee AJ, Mian F, Poznanski SM, Stackaruk M, Chan T, Chew MV, et al. Type I Interferon Receptor on NK Cells Negatively Regulates Interferon$\gamma$ Production. Front Immunol (2019) 10:1261. doi: 10.3389/fimmu. 2019.01261

85. Zhou Q, Niño DF, Yamaguchi Y, Wang S, Fulton WB, Jia H, et al. Necrotizing Enterocolitis Induces T Lymphocyte-Mediated Injury in the Developing Mammalian Brain. Sci Transl Med (2021) 13:1-13. doi: 10.1126/ SCITRANSLMED.AAY6621

86. Dandekar AA, Anghelina D, Perlman S. Bystander CD8 T-Cell-Mediated Demyelination is Interferon- $\gamma$-Dependent in a Coronavirus Model of Multiple Sclerosis. Am J Pathol (2004) 164:363-9. doi: 10.1016/S0002-9440 (10)63126-4

87. Iijima N, Thompson JM, Iwasaki A. Dendritic Cells and Macrophages in the Genitourinary Tract. Mucosal Immunol (2008) 1:451-9. doi: 10.1038/mi.2008.57

88. Longhi MP, Trumpfheller C, Idoyaga J, Caskey M, Matos I, Kluger C, et al. Dendritic Cells Require a Systemic Type I Interferon Response to Mature and Induce CD4+ Th1 Immunity With Poly IC as Adjuvant. J Exp Med (2009) 206:1589-602. doi: 10.1084/jem.20090247

89. Zhao X, Deak E, Soderberg K, Linehan M, Spezzano D, Zhu J, et al. Vaginal Submucosal Dendritic Cells, But Not Langerhans Cells, Induce Protective Th1 Responses to Herpes Simplex Virus-2. J Exp Med (2003) 197:153-62. doi: $10.1084 /$ jem.20021109

90. Lee AG, Scott JM, Fabbrizi MR, Jiang X, Sojka DK, Miller MJ, et al. T Cell Response Kinetics Determines Neuroinfection Outcomes During Murine HSV Infection. JCI Insight (2020) 5:1-17. doi: 10.1172/jci.insight.134258

91. Johnson AJ, Nelson MH, Bird MD, Chu CF, Milligan GN. Herpes Simplex Virus (HSV)-Specific T Cells Activated in the Absence of IFN-Gamma Express Alternative Effector Functions But Are Not Protective Against Genital HSV-2 Infection. J Reprod Immunol (2010) 84:8-15. doi: 10.1016/ j.jri.2009.09.007

92. Chen B, Lee AJ, Chew MV, Ashkar AA. NK Cells Require Antigen-Specific Memory CD4 + T Cells to Mediate Superior Effector Functions During HSV-2 Recall Responses In Vitro. J Leukoc Biol (2017) 101:1045-52. doi: 10.1189/jlb.4a0416-192r

93. Le Bon A, Thompson C, Kamphuis E, Durand V, Rossmann C, Kalinke U, et al. Cutting Edge: Enhancement of Antibody Responses Through Direct Stimulation of B and T Cells by Type I IFN. J Immunol (2006) 176:2074-8. doi: 10.4049/jimmunol.176.4.2074

94. Kolumam GA, Thomas S, Thompson LJ, Sprent J, Murali-Krishna K. Type I Interferons Act Directly on CD8 T Cells to Allow Clonal Expansion and Memory Formation in Response to Viral Infection. J Exp Med (2005) 202:637-50. doi: 10.1084/jem.20050821

95. Kohlmeier JE, Cookenham T, Roberts AD, Miller SC, Woodland DL. Type I Interferons Regulate Cytolytic Activity of Memory CD8+ T Cells in the Lung Airways During Respiratory Virus Challenge. Immunity (2010) 33:96-105. doi: 10.1016/j.immuni.2010.06.016 
96. Wang Y, Swiecki M, Cella M, Alber G, Schreiber RD, Gilfillan S, et al. Timing and Magnitude of Type I Interferon Responses by Distinct Sensors Impact CD8 T Cell Exhaustion and Chronic Viral Infection. Cell Host Microbe (2012) 11:631-42. doi: 10.1016/j.chom.2012.05.003

97. McNally JM, Zarozinski CC, Lin M-Y, Brehm MA, Chen HD, Welsh RM. Attrition of Bystander CD8 T Cells During Virus-Induced T-Cell and Interferon Responses. J Virol (2001) 75:5965-76. doi: 10.1128/ jvi.75.13.5965-5976.2001

98. Bahl K, Kim S-K, Calcagno C, Ghersi D, Puzone R, Celada F, et al. IFNInduced Attrition of CD8 T Cells in the Presence or Absence of Cognate Antigen During the Early Stages of Viral Infections. J Immunol (2006) 176:4284-95. doi: 10.4049/jimmunol.176.7.4284

99. Marshall HD, Urban SL, Welsh RM. Virus-Induced Transient Immune Suppression and the Inhibition of T Cell Proliferation by Type I Interferon. J Virol (2011) 85:5929-39. doi: 10.1128/jvi.02516-10

100. Harandi AM, Svennerholm B, Holmgren J, Eriksson K. Protective Vaccination Against Genital Herpes Simplex Virus Type 2 (HSV-2) Infection in Mice is Associated With a Rapid Induction of Local IFN- $\gamma$ Dependent RANTES Production Following a Vaginal Viral Challenge. Am J Reprod Immunol (2001) 46:420-4. doi: 10.1034/j.1600-0897.2001.d01-34.x

101. Iijima N, Iwasaki A. A Local Macrophage Chemokine Network Sustains Protective Tissue-Resident Memory CD4 T Cells. Sci (80- ) (2014) 346:93-8. doi: 10.1126/science. 1257530

102. Chetty A, Darby MG, Vornewald PM, Martín-Alonso M, Filz A, Ritter M, et al. Il4ra-Independent Vaginal Eosinophil Accumulation Following Helminth Infection Exacerbates Epithelial Ulcerative Pathology of HSV-2 Infection. Cell Host Microbe (2021) 29:579-93.e5. doi: 10.1016/j.chom. 2021.02.004

103. Ford ES, Sholukh AM, Boytz R, Carmack SS, Klock A, Phasouk K, et al. B Cells, Antibody-Secreting Cells, and Virus-Specific Antibodies Respond to Herpes Simplex Virus 2 Reactivation in Skin. J Clin Invest (2021) 131:1-12. doi: $10.1172 /$ JCI1 42088

104. Parr MB, Parr EL. Immunity to Vaginal Herpes Simplex Virus- 2 Infection in B-Cell Knockout Mice. Immunology (2000) 101:126-31. doi: 10.1046/j.13652567.2000.00080.x

105. Halford WP, Geltz J, Messer RJ, Hasenkrug KJ. Antibodies Are Required for Complete Vaccine-Induced Protection Against Herpes Simplex Virus 2. PloS One (2015) 10:1-26. doi: 10.1371/journal.pone.0145228

106. Fink K, Lang KS, Manjarrez-Orduno N, Junt T, Senn BM, Holdener M, et al. Early Type I Interferon-Mediated Signals on B Cells Specifically Enhance Antiviral Humoral Responses. Eur J Immunol (2006) 36:2094-105. doi: $10.1002 /$ eji.200635993

107. Le Bon A, Schiavoni G, D’Agostino G, Gresser I, Belardelli F, Tough DF. Type I Interferons Potently Enhance Humoral Immunity and can Promote Isotype Switching by Stimulating Dendritic Cells In Vivo. Immunity (2001) 14:461-70. doi: 10.1016/S1074-7613(01)00126-1

108. Jego G, Palucka AK, Blanck JP, Chalouni C, Pascual V, Banchereau J. Plasmacytoid Dendritic Cells Induce Plasma Cell Differentiation Through Type I Interferon and Interleukin 6. Immunity (2003) 19:225-34. doi: 10.1016/S1074-7613(03)00208-5

109. Bekeredjian-Ding IB, Wagner M, Hornung V, Giese T, Schnurr M, Endres S, et al. Plasmacytoid Dendritic Cells Control TLR7 Sensitivity of Naive B Cells via Type I IFN. J Immunol (2005) 174:4043-50. doi: 10.4049/ jimmunol.174.7.4043

110. Oh JE, Iijima N, Song E, Lu P, Klein J, Jiang R, et al. Migrant Memory B Cells Secrete Luminal Antibody in the Vagina. Nature (2019) 571:122-6. doi: 10.1038/s41586-019-1285-1

111. Iijima N, Iwasaki A. Access of Protective Antiviral Antibody to Neuronal Tissues Requires CD4 T-Cell Help. Nature (2016) 533:552-6. doi: 10.1038/nature17979

112. Stanberry LR, Spruance SL, Cunningham AL, Bernstein DI, Mindel A, Sacks S, et al. Glycoprotein-D-Adjuvant Vaccine to Prevent Genital Herpes. N Engl $J$ Med (2002) 347:1652-61. doi: 10.1056/nejmoa011915
113. Straus SE, Wald A, Kost RG, McKenzie R, Langenberg AGM, Hohman P, et al. Immunotherapy of Recurrent Genital Herpes With Recombinant Herpes Simplex Virus Type 2 Glycoproteins D and B: Results of a Placebo-Controlled Vaccine Trial. J Infect Dis (1997) 176:1129-34. doi: $10.1086 / 514103$

114. Belshe RB, Leone PA, Bernstein DI, Wald A, Levin MJ, Stapleton JT, et al. Efficacy Results of a Trial of a Herpes Simplex Vaccine. N Engl J Med (2012) 366:34-43. doi: 10.1056/NEJMoa1103151

115. De Bruyn G, Vargas-Cortez M, Warren T, Tyring SK, Fife KH, Lalezari J, et al. A Randomized Controlled Trial of a Replication Defective ( $\mathrm{gH}$ Deletion) Herpes Simplex Virus Vaccine for the Treatment of Recurrent Genital Herpes Among Immunocompetent Subjects. Vaccine (2006) 24:91420. doi: $10.1016 /$ j.vaccine.2005.08.088

116. Cattamanchi A, Posavad CM, Wald A, Baine Y, Moses J, Higgins TJ, et al. Phase I Study of a Herpes Simplex Virus Type 2 (HSV-2) DNA Vaccine Administered to Healthy, HSV-2-Seronegative Adults by a Needle-Free Injection System. Clin Vaccine Immunol (2008) 15:1638-43. doi: 10.1128/CVI.00167-08

117. Gilbert PB, Excler JL, Tomaras GD, Carpp LN, Haynes BF, Liao HX, et al. Antibody to HSV gD Peptide Induced by Vaccination Does Not Protect Against HSV-2 Infection in HSV-2 Seronegative Women. PloS One (2017) 12:1-15. doi: 10.1371/journal.pone.0176428

118. Bernstein DI, Aoki FY, Tyring SK, Stanberry LR, St.-Pierre C, Shafran SD, et al. Safety and Immunogenicity of Glycoprotein D-Adjuvant Genital Herpes Vaccine. Clin Infect Dis (2005) 40:1271-81. doi: 10.1086/429240

119. Svensson A, Bellner L, Magnusson M, Eriksson K. Role of IFN- $\alpha / \beta$ Signaling in the Prevention of Genital Herpes Virus Type 2 Infection. J Reprod Immunol (2007) 74:114-23. doi: 10.1016/j.jri.2006.09.002

120. Peng T, Zhu J, Klock A, Phasouk K, Huang M-L, Koelle DM, et al. Evasion of the Mucosal Innate Immune System by Herpes Simplex Virus Type 2. J Virol (2009) 83:12559-68. doi: 10.1128/jvi.00939-09

121. Zhou Y, Wang Z, Xu Y, Zhang Z, Hua R, Liu W, et al. Optimized DNA Vaccine Enhanced by Adjuvant IL28B Induces Protective Immune Responses Against Herpes Simplex Virus Type 2 in Mice. Viral Immunol (2017) 30:601-14. doi: 10.1089/vim.2017.0033

122. Davidson S, Crotta S, McCabe TM, Wack A. Pathogenic Potential of Interferon $\alpha \beta$ in Acute Influenza Infection. Nat Commun (2014) 5:3864. doi: $10.1038 /$ ncomms 4864

123. Galani IE, Triantafyllia V, Eleminiadou EE, Koltsida O, Stavropoulos A, Manioudaki $M$, et al. Interferon- $\lambda$ Mediates Non-Redundant Front-Line Antiviral Protection Against Influenza Virus Infection Without Compromising Host Fitness. Immunity (2017) 46:875-890.e6. doi: 10.1016/j.immuni.2017.04.025

124. Feld JJ, Kandel C, Biondi MJ, Kozak RA, Zahoor MA, Lemieux C, et al. Peginterferon Lambda for the Treatment of Outpatients With COVID-19: A Phase 2, Placebo-Controlled Randomised Trial. Lancet Respir Med (2021) 2600:1-13. doi: 10.1016/s2213-2600(20)30566-x

Conflict of Interest: The authors declare that the research was conducted in the absence of any commercial or financial relationships that could be construed as a potential conflict of interest.

Publisher's Note: All claims expressed in this article are solely those of the authors and do not necessarily represent those of their affiliated organizations, or those of the publisher, the editors and the reviewers. Any product that may be evaluated in this article, or claim that may be made by its manufacturer, is not guaranteed or endorsed by the publisher.

Copyright $\odot 2021$ Feng, Balint, Vahedi and Ashkar. This is an open-access article distributed under the terms of the Creative Commons Attribution License (CC BY). The use, distribution or reproduction in other forums is permitted, provided the original author(s) and the copyright owner(s) are credited and that the original publication in this journal is cited, in accordance with accepted academic practice. No use, distribution or reproduction is permitted which does not comply with these terms. 